\title{
ECG Coding by Wavelet-Based Linear Prediction
}

\author{
A. G. Ramakrishnan,* Senior Member, IEEE, and Supratim Saha, Student Member, IEEE
}

\begin{abstract}
This paper presents a novel coding scheme for electrocardiogram (ECG). Following beat delineation, the periods of the beats are normalized by multirate processing. After amplitude normalization, discrete wavelet transform is applied to each beat. Due to the period and amplitude normalization, the wavelet transform coefficients bear a high correlation across beats at identical locations. To increase the compression ratio, the residual sequence obtained after linear prediction of the significant wavelet coefficients is transmitted to the decoder. The difference between the actual period and the mean beat period, and that between the actual scale factor and the average amplitude scale factor are also transmitted for each beat. At the decoder, the inverse wavelet transform is computed from the reconstructed wavelet transform coefficients. The original amplitude and period of each beat are then recovered. The approximation achieved, at an average rate of $180 \mathrm{~b} / \mathrm{s}$, is of high quality. We have evaluated the normalized maximum amplitude error and its position in each cycle, in addition to the normalized root mean square error. The significant feature of the proposed technique is that, while the error is nearly uniform throughout the cycle, the diagnostically crucial QRS region is kept free of maximal reconstruction error.
\end{abstract}

Index Terms - Cyclostationarity, discrete wavelet transform, ECG compression, multirate processing, period normalization.

\section{INTRODUCTION}

$\mathbf{E}$ LECTROCARDIOGRAM (ECG) coding is required in several applications such as ambulatory monitoring, patient data bases, medical education systems, and transmission over telephone lines. ECG is oscillatory in nature, although not periodic in the strict mathematical sense. Looking at the time evolution of this signal, we can observe a concatenation of similar events or periods, which almost never reproduce themselves identically. For the same subject, the cycle-tocycle variation in the beat period is, in general, much higher than the occasional variations in the amplitude, position, and width of the constituent waves. However, most techniques of ECG compression reported till now have not exploited this correlation between cycles (interbeat correlation). There is also some redundancy within each ECG cycle. Direct time-domain techniques such as [1]-[3], and transform-domain techniques such as [4]-[8] have considered only this intrabeat correlation between successive samples. Whereas, long term prediction [9] and average beat subtraction [10] techniques have used only the beat to beat correlation, ignoring the redundancy

Manuscript received January 31, 1996; revised July 8, 1997. Asterisk indicates corresponding author.

*A. G. Ramakrishnan is with the Department of Electrical Engineering, Indian Institute of Science, Bangalore 560012 India (e-mail: ramkiag@ee.iisc.ernet.in).

S. Saha is with the Department of Electrical Engineering, Indian Institute of Science, Bangalore 560012 India.

Publisher Item Identifier S 0018-9294(97)07601-5. within beat. Another limitation of [9] and [10] rises from the fact that, since the period of a beat changes constantly, points that are equidistant and farther from the $R$ wave in two different cycles are not always well correlated. Further, [9] requires detection of the end points of each cycle, in addition to QRS detection and the correlation of QRS complex of each beat with a codebook of complexes. Similarly, the methods based on modeling, such as [11] and [12] require component identification for both model order selection and proper cycle separation. Parametric techniques [12] have minimized only the intrabeat redundancy and not the other. The technique in [11] exploits both, but the authors clearly say that heart rate variability makes it difficult to assign a fixed number of bits to the differences in model parameters.

The technique proposed in this paper accounts for the variations in the beat periods and then exploits both the intercycle and the intracycle correlations. The method requires the detection of no component other than the $R$-wave. We first make the period of each beat constant by a nearly reversible transformation, in which the original periods can be restored without any loss. We perform amplitude normalization on these period-normalized beats. On these "period and amplitude normalized (PAN) beats," discrete wavelet transform (DWT) is applied. Linear prediction of selected wavelet coefficients is then performed and only the prediction error sequence is transmitted.

\section{Period AND Amplitude Normalization}

For delineating cycles, we define a cycle as the signal from one $R$-wave to the next. We used the technique reported in [13] for QRS detection. We normalize the period of each isolated beat by multirate techniques [14]. This involves sampling rate change by different fractional factors for different cycles. This converts the beats of differing periods into beats of a constant period, thus eliminating the effect of heart rate variability. The fixed length of the cycles is selected based on the maximum possible period of any cardiac cycle and the sampling frequency. The modified sampling rate must still satisfy the Nyquist criterion. We have selected a length such that the new sampling rate is always higher than the original one, ensuring that there will be no distortion of the signal. The mean beat period (MBP) is estimated from some initial cycles of the data being coded. This value is initially sent to the decoder. During encoding, the difference between the actual period of a cycle and the MBP is transmitted.

To perform period normalization, we first interpolate the variable period beat vectors by a factor $L$, which is the fixed period planned for. Then the signal is downsampled by the appropriate factor for each cycle, so that the length of all cycles 


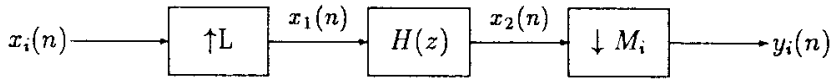

Fig. 1. Period normalization.

becomes uniform. In our case, only the interpolation filter and downsampling are required. Since ECG is a highly correlated signal, and since it has been interpolated by a sufficiently high value, no error occurs in downsampling. The details of implementation are given below.

If $x(n)$ is the input to an interpolation filter with an upsampling factor $L$ and an impulse response $h(n)$, then the output $y(n)$ is given by

$$
y(n)=\sum_{k=-\infty}^{\infty} x(k) h(n-k L) .
$$

The upsampler simply inserts $L-1$ zeros between successive samples. The filter $h(n)$, which operates at a rate $L$ times higher than that of the input signal, replaces the inserted zeros with interpolated values. Polyphase implementation of this filter [14] ensures efficient interpolation. The output $y(n)$ of a decimation filter, with an impulse response $h(n)$ and a downsampling factor $M$, is given by

$$
y(n)=\sum_{k=-\infty}^{\infty} x(k) h(n M-k)
$$

where $h(n)$ is a lowpass filter used to remove the aliasing caused by the downsampling of the signal. In case the signal does not contain frequencies above $\pi / M$, there is no need for the decimation filter; downsampling alone will do. The change of sampling rate thus achieved is a reversible process, provided Nyquist condition is satisfied; if the resampled beat is brought back to the original sampling rate by multirate processing, there will be no distortion. The output of our system is given by

$$
\mathbf{Y}_{i}(n)=\sum_{k=0}^{P_{i}-1} \mathbf{X}_{i}(k) h\left(n M_{i}-k L\right)
$$

where $\mathbf{X}_{i}(n), \mathbf{Y}_{i}(n)$ are the $n$th samples of the $i$ th input beat and output PAN beat, respectively, $h(n)$ is the impulse response of the filter, $P_{i}$ is the total number of samples in $i$ th original beat, and $L, M_{i}$ are, respectively, the upsampling and downsampling factors for the $i$ th beat vector. The block schematic for this operation is shown in Fig. 1. The interpolation is efficiently accomplished in multiple stages as shown in Fig. 2. (cf., [14]).

Amplitude normalization brings about further similarity between the beat patterns. Each sample of a beat is divided by the magnitude of the largest sample of that beat. This makes the highest amplitude sample(s) of each beat equal to unity. Thus, the variations between the magnitudes of different cycles are minimized. Fig. 3 shows that PAN does not introduce any distortion in the signal and also demonstrates its efficacy in enhancing the interbeat correlation. To begin with, the average amplitude scale factor (AASF) is obtained from a few initial beats and is sent to the decoder. Subsequently, for each cycle being coded, the difference between the maximum amplitude of that cycle and the AASF is transmitted to the decoder.

\section{WAVELET-BASEd LinEAR PREDICTION OF PAN BEATS}

In any ECG waveform, the QRS complex is a welllocalized, high-frequency region. The $P$ and $T$ waves are low-frequency components, and the PQ and TP segments are nearly isoelectric with limited information. The ST segment is a very low-frequency, time-localized component. These time-localized components affect the entire spectrum of the ECG, and hence, the Fourier transform is not quite adequate to characterize it. In fact, accurate representation of such localized components would require a linear combination of a large number of sine and cosine waves. Conventional transforms [such as Karhunen-Loeve transform (KLT), discrete cosine transform (DCT), or DFT] can perform well only when the signal is stationary, and the energy is exclusively concentrated in certain bands. Thus, coding schemes based on such transforms have not really performed well. Reference [15] used discrete Legendre polynomials to account for the nonstationarity of ECG beats and obtained better results. However, wavelets have a lot of potential for the representation of nonstationary signals. Thus, the ECG signal, being highly nonstationary within each beat, lends itself quite well to wavelet transform-based coding. Senhadji et al. [16] first proposed the use of wavelets for the analysis of ECG. Thakor [17] and Bradie [18] applied DWT for ECG compression, but neither attempted to make use of the interbeat correlation.

\section{A. Wavelet Transform for Signal Decomposition}

In wavelet analysis, a mother function $\Psi(x)$ and a linear combination of its dilated and/or shifted versions are used to represent a given signal

$$
f(x)=\sum_{j} \sum_{k} w_{j, k} \Psi_{j, k}(x)
$$

where $f(x)$ is the signal to be analyzed, $\Psi_{j, k}(x)$ is the dilated and shifted version of mother wavelet $\Psi(x), j, k \in Z$, and determine the dilation and shift factor, respectively, $w_{j, k}$ are the wavelet coefficients, and

$$
\Psi_{j, k}(x)=\Psi\left(2^{j} x-k\right) .
$$

It is desired that the wavelet basis functions be orthonormal [19] in order to simplify the computation of the coefficients. From (4) and orthonormality of basis functions, we get the wavelet coefficients $w_{j k}$ as

$$
w_{j, k}=\left\langle f(x), \Psi_{j, k}(x)\right\rangle
$$

or

$$
w_{j, k}=\int_{-\infty}^{+\infty} f(x) \Psi_{j, k}(x) d x .
$$

From (4) and (5) we get

$$
f(x)=\sum_{j} \sum_{k} w_{j, k} \Psi\left(2^{j} x-k\right) .
$$




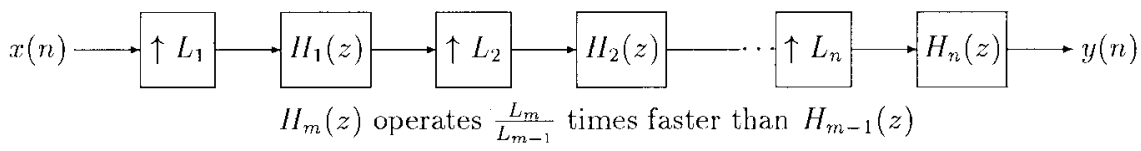

Fig. 2. Multistage implementation of interpolation.

(a)

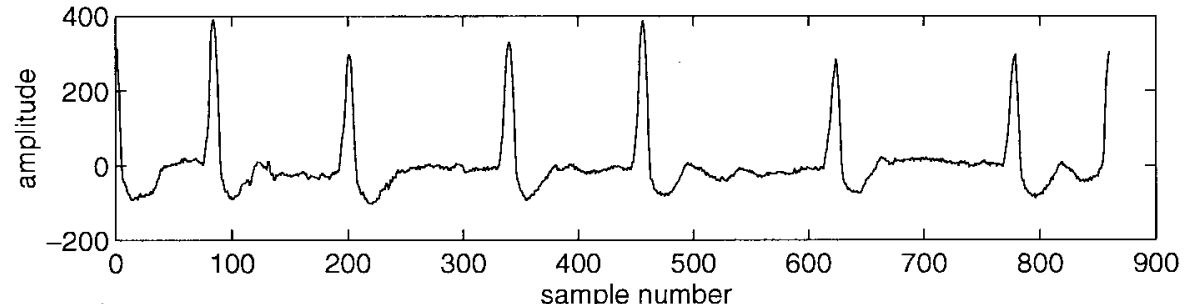

(b)

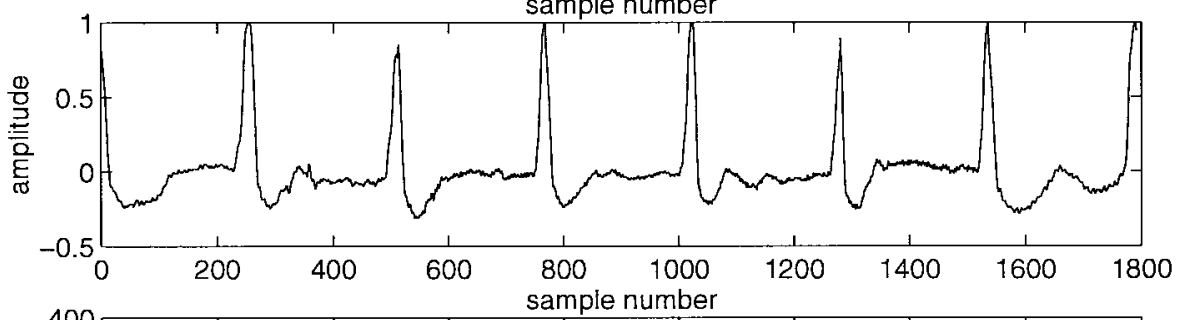

(c)

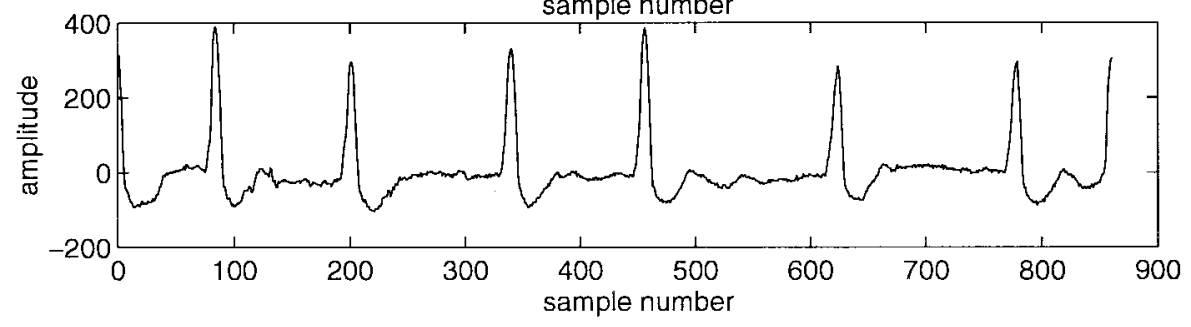

Fig. 3. PAN: A nearly distortion-free transformation: (a) Original beats, (b) PAN beats, and (c) reconstructed beats.

While setting up a discrete wavelet transform algorithm, it is convenient to limit the range of the independent variable $x$ to one unit interval so that $f(x)$ is defined only for $0 \leq$ $x \leq 1$ [20]. Here, $x$ is a nondimensional variable; so if the independent variable is time $t$, and we are interested in a signal over duration $T$, then $x=t / T$.

Of the many available orthogonal basis functions $\Psi_{j, k}(x) \epsilon$ $\mathbf{L}^{2}(\mathbf{R})$, we have used Daubechies-4 (D4) functions [21] for representing each PAN beat. The D4 wavelet $\Psi_{0,0}(x)$ occupies three unit intervals $0 \leq x \leq 3$. The expansion of $f(x)$ in $0 \leq x \leq 1$ can be written [20] as

$$
\begin{aligned}
f(x)= & w_{01} \phi(x)+w_{11} \Psi(x)+\left[\begin{array}{ll}
w_{21} & w_{22}
\end{array}\right]\left[\begin{array}{c}
\Psi(2 x) \\
\Psi(2 x-1)
\end{array}\right] \\
& +\left[\begin{array}{llll}
w_{31} & w_{32} & w_{33} & w_{34}
\end{array}\right]\left[\begin{array}{c}
\Psi(4 x) \\
\Psi(4 x-1) \\
\Psi(4 x-2) \\
\Psi(4 x-3)
\end{array}\right]+\cdots
\end{aligned}
$$

where $\phi(x)=1,0 \leq x<1$ and $w_{j k}$ refers to the wavelet coefficient at scale $j$ and location $k$. Let us form a vector $X$ by stacking the wavelet coefficients at scales $0,1,2, \ldots, 7$ as defined below

$$
X=\left[\begin{array}{lllllllll}
w_{01} & w_{11} & w_{21} & w_{22} & w_{31} & w_{32} & w_{33} & w_{34} & \cdots
\end{array}\right]
$$

Then, from (9) and (10) we get

$$
\begin{aligned}
f(x)= & X(0) \phi(x)+X(1) \Psi(x) \\
& +[X(2) X(3)]\left[\begin{array}{c}
\Psi(2 x) \\
\Psi(2 x-1)
\end{array}\right] \\
& +[X(4) X(5) X(6) X(7)]\left[\begin{array}{c}
\Psi(4 x-1) \\
\Psi(4 x-2) \\
\Psi(4 x-3)
\end{array}\right] \\
& +\cdots+X\left(2^{j}+k\right) \Psi\left(2^{j} x-k\right)
\end{aligned}
$$

where $X(j)$ is the $j$ th element of the vector $X$.

To compute the wavelet coefficients for the signal (sampled at equally spaced intervals in $0 \leq x \leq 1$ ) of each PAN beat, we use Mallat's pyramidal DWT algorithm [22], which requires that the number of samples in the sequence be a power of 2 . The normalized beat is 256 samples long and therefore, DWT entails an eight level dyadic decomposition. Here, each beat is shifted to the origin before it is processed, since DWT is not time-shift invariant. If the above transform is applied to a PAN beat, owing to the nonstationary nature of the latter and because of nonuniform distribution of energy across the different scales, it turns out that not all the wavelet coefficients are required for a reasonable quality of reconstruction. It has been observed that only the highest $20 \%$ of the coefficients are necessary for reconstruction without loss of any significant 
rhythm or morphological information. Thus, an intermediate compression of roughly $5: 1$ is achieved by retaining only some (in fact, a chosen set as explained later) of the wavelet coefficients and neglecting the rest.

\section{B. Linear Prediction of DWT Coefficients}

Since there is a definite correlation between the corresponding wavelet coefficients of different normalized cycles, the current one can be estimated from a certain number of past coefficients, and only the residual need be transmitted. This, precisely, is the principle of linear prediction (LP), using linear mean squared criterion. Ruttiman and Pipberger applied LP on raw ECG samples without any preprocessing and obtained a reduction in the variance [23]. However, we perform LP of wavelet coefficients across beats, at corresponding scale and location. The variance of the residuals obtained is less than that of the original coefficients. Thus, we are able to allocate three bits less to each residual than the number of bits required for each wavelet coefficient.

The wavelet coefficient for the $i$ th beat at scale $j$ and location $n, w_{j n}(i)$ is estimated by

$$
\begin{aligned}
\hat{w}_{j n}(i)= & a_{1, j n} w_{j n}(i-1)+a_{2, j n} w_{j n}(i-2)+\cdots \\
& +a_{p, j n} w_{j n}(i-p)
\end{aligned}
$$

where $p$ is the order of prediction and $a_{1, j n}, \cdots, a_{p, j n}$ are the LP coefficients. The prediction error for scale $j$ and location $n$ is then given by

$$
e_{j n}(i)=w_{j n}(i)-\hat{w}_{j n}(i)
$$

The LP parameters in (12) are chosen according to minimum mean square error criteria, which is equivalent to the orthogonality of the error with respect to the data used in the prediction [24].

We stack the wavelet coefficients at all the scales for each PAN beat as a vector. These vectors concatenated together form a near-cyclostationary sequence. This occurs despite the fact that DWT is time-shift invariant, since the beats are shifted to origin. Let $\mathbf{X}_{j}$ be the vector formed by all the wavelet coefficients of the $j$ th PAN beat [refer (10)]. i.e.,

$$
\begin{aligned}
X_{j}= & {\left[w_{01}(j) w_{11}(j) w_{21}(j) w_{22}(j) w_{31}(j)\right.} \\
& \left.\cdot w_{32}(j) w_{33}(j) w_{34}(j) \cdots\right] .
\end{aligned}
$$

Then

$$
\mathbf{V}=\left[X_{1} X_{2} \cdots X_{N_{T}}\right]
$$

is the near-cyclostationary sequence, where $N_{T}$ is the total number of beats being processed. Components of $\mathbf{V}$ nearly satisfy the wide-sense cyclostationary conditions, viz., the mean, $\mu_{v(n)}=E[v(n)] \sim E[v(n+N)]$, where $N$ is the period and $R_{v}\left(n_{1}+k N, n_{2}+k N\right) \sim R_{v}\left(n_{1}, n_{2}\right)$ for any integer $k$.

From this sequence, independent linear predictions are performed on the following data sets, each of which is formed by grouping the corresponding coefficients from all the beats

$$
\begin{aligned}
U_{0}= & \left\{\mathbf{V}(\mathbf{0}), \mathbf{V}(\mathbf{N}), \mathbf{V}(\mathbf{2} \mathbf{N}), \cdots, \mathbf{V}\left[\left(\mathbf{N}_{\mathbf{T}}-\mathbf{1}\right) \mathbf{N}\right]\right\} \\
U_{1}= & \{\mathbf{V}(\mathbf{1}), \mathbf{V}(\mathbf{N}+\mathbf{1}), \mathbf{V}(\mathbf{2} \mathbf{N}+\mathbf{1}), \cdots \\
& \left.\mathbf{V}\left[\left(\mathbf{N}_{\mathbf{T}}-\mathbf{1}\right) \mathbf{N}+\mathbf{1}\right]\right\} \\
\vdots & \vdots \\
U_{N-1}= & \{\mathbf{V}(\mathbf{N}-\mathbf{1}), \mathbf{V}(\mathbf{2} \mathbf{N}-\mathbf{1}), \cdots \\
& \left.\mathbf{V}\left[\left(\mathbf{N}_{\mathbf{T}}-\mathbf{1}\right) \mathbf{N}+\mathbf{N}-\mathbf{1}\right]\right\} .
\end{aligned}
$$

Because of the near-wide sense stationary nature of these individual sets, the use of time-averaged autocorrelation, which depends only on the lag, is justified. The above LP enables us to transmit only the residual wavelet coefficients, and with a smaller number of bits.

\section{Choice of Significant Wavelet Coefficients}

The fact that not all the wavelet coefficients are significant in the reconstruction of any beat, is because of the high suitability of time-localized basis functions for representing the locally nonstationary ECG cycle. By choosing a fixed set of significant coefficients to be transmitted from each beat, we can eliminate the overhead of bits required to send the positions of the coefficients retained in each beat. This, however, needs to be done without losing important rhythm and morphological information. PAN beats enable us to achieve this end. We choose the $N_{c}$ highest amplitude wavelet coefficients from each of the first $K$ PAN beats, and keep their locations in different sets $P_{i}, i=1,2, \cdots, K$, in descending order of significance. The algorithm used for deciding the set of locations of significant wavelet coefficients to be retained in each cycle follows.

1) $P_{i}=\left\{p_{i j}\right\}, i=1,2, \cdots, K ; j=1,2, \cdots, N_{c} ; N_{c} \leq$ $N_{R}$ where $N_{R}$ is the final number of coefficients to be retained in each beat.

2) Initialize $N=N_{m} \leq N_{c}$, where $N_{m}$ is the minimum number of highest amplitude wavelet coefficients to be retained in each beat.

3) $P_{i}^{N}=\left\{p_{i j}\right\}, i=1,2, \cdots, K ; j=1,2, \cdots, N$.

4) $S_{N}=\bigcup_{i=1}^{K} P_{i}^{N}$.

5) If $\left|S_{N}\right|=N_{R}$, stop.

6) If $\left|S_{N}\right|<N_{R}, N=N+1$ and go to Step 4 .

7) If $\left|S_{N}\right|>N_{R}$, then from the last set of locations added, remove $\left(\left|S_{N}\right|-N_{R}\right)$ entries corresponding to coefficients of least magnitude.

8) $S_{N}$ is the set of locations of wavelet coefficients to be retained.

Thus, the selection ensures that at least the first $N-1$ highest-amplitude coefficients are retained in each cycle, $N_{m} \leq N-1 \leq N_{R}$. The results reported in this paper have been obtained using $N_{m}=30, N_{R}=50$, and $K=15$. Thus LP needs to be performed only for the sets $U_{i}, i \in S_{N}$. The block schematic for the encoder is shown in Fig. 4.

\section{Beat Reconstruction}

The block schematic of the decoder is shown in Fig. 5. The received residual coefficients are processed by independent 


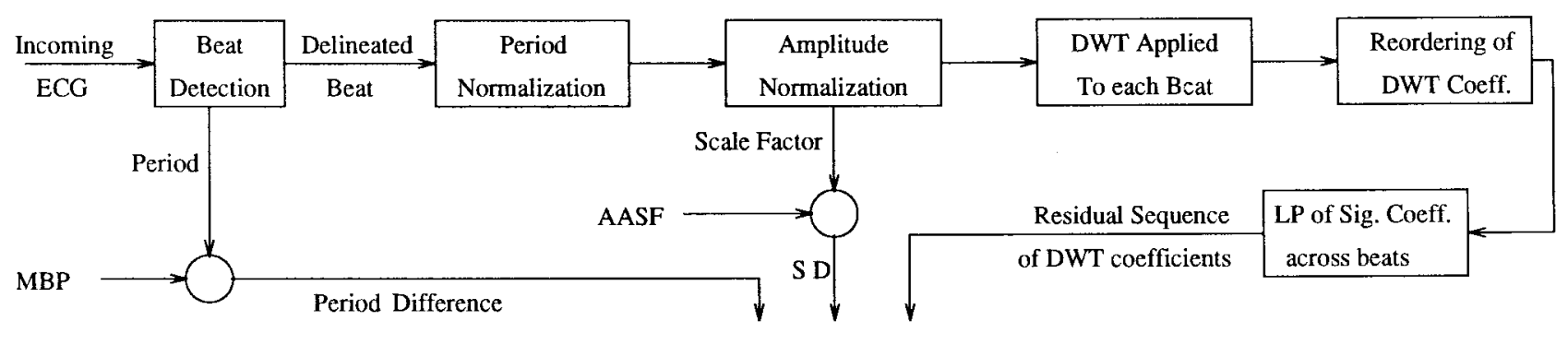

Transmit / Store

Fig. 4. Block schematic of the encoder.

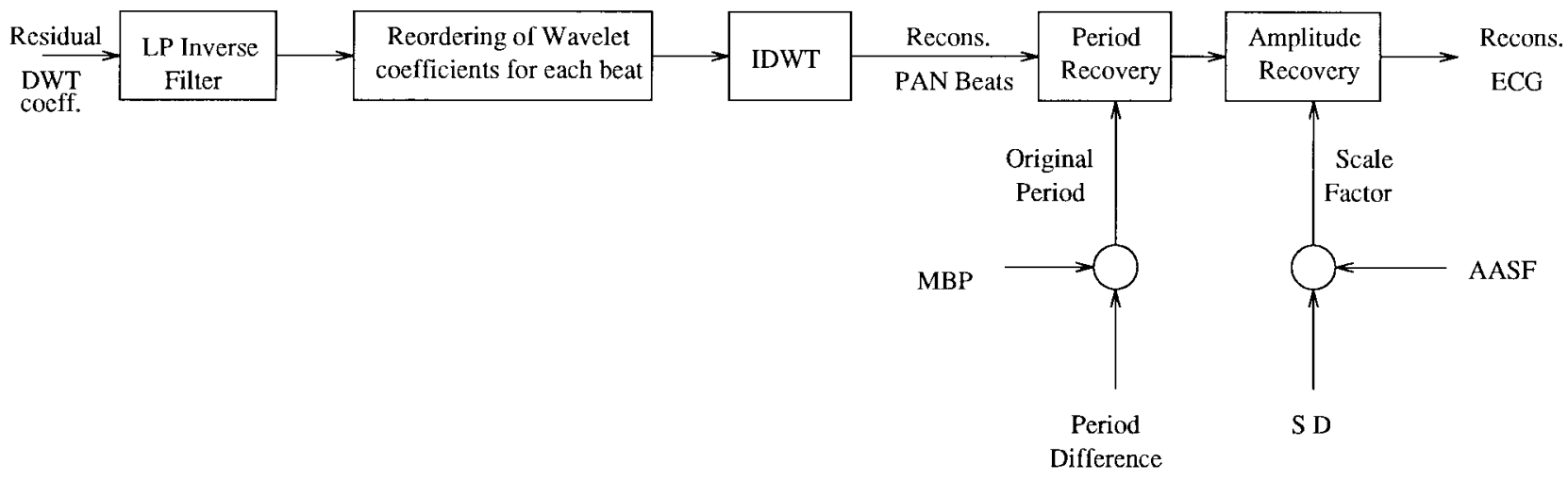

Fig. 5. Block schematic of the decoder.

inverse filters, whose impulse response depends only on the respective forward LP filters. The reconstructed coefficients across the PAN ECG beats are reordered to get the DWT coefficients for each beat. Inverse DWT of these coefficients is computed to obtain the reconstructed PAN beats. The actual period of each cycle is obtained from the period difference and the MBP. The original period beat is then recovered from the reconstructed cycle by the method discussed in Section II with appropriate parameter changes. The original scale factor is obtained from the scale factor difference and the AASF. The period-recovered beats are then multiplied by the corresponding scale factors to get the reconstructed beats, and consequently, the reconstructed ECG.

\section{RESUlTS AND Discussion}

The proposed method was tested on ECG data obtained from a hospital. The signal was sampled at $250 \mathrm{~Hz}$ and quantized with 12-b resolution. During period normalization, the length of each cycle was changed to 256 samples. The technique was also applied on some abnormal data from the Massachusetts Institute of Technology (MIT) database. Figs. 6-8 give the original, reconstructed, and the error waveforms, respectively for three of the subjects. Fig. 6 shows the performance of our technique on an arrhythmic data and one can clearly notice that the technique really performs very well with variable interbeat intervals. Figs. 7 and 8 show the results on other types of data.

The performance of the method is evaluated using the measures discussed below. The compression ratio (CR) has been computed as follows:

$$
\begin{aligned}
& \mathrm{CR}= \\
& \frac{K \sum_{i=1}^{N_{T}} T_{i}}{N_{T}\left(N_{R} b+\alpha_{a}+\alpha_{p}\right)+\left\{N_{R}\left(p b_{p}+b_{n z}\right)+b_{a p}+b_{a a}\right\}}
\end{aligned}
$$

where $K$ is the number of $\mathrm{b} / \mathrm{sample}$ in the original signal, $T_{i}$ is the period of $i$ th beat, $N_{T}$ is the total number of beats, $N_{R}$ is the number of coefficients whose residuals are transmitted for each cycle, $b, \alpha_{a}$, and $\alpha_{p}$ are the number of bits used for transmitting each residual, scale factor difference, and period difference, respectively, $p$ is the order of the LP filter, $b_{p}, b_{n z}$, $b_{a p}$, and $b_{a a}$ are the number of bits used for transmitting each LP parameter, each element of $S_{N}$, the MBP, and the AASF, respectively. The terms within braces in the denominator need to be sent to the decoder only once.

\section{A. Normalized Root Mean Square Error (NRMSE)}

The expression for this common error measure is

$$
\text { NRMSE }=\sqrt{\frac{\sum_{i=0}^{N-1}\left[x_{o}(i)-x_{r}(i)\right]^{2}}{\sum_{i=0}^{N-1} x_{o}^{2}(i)}}
$$


(a)
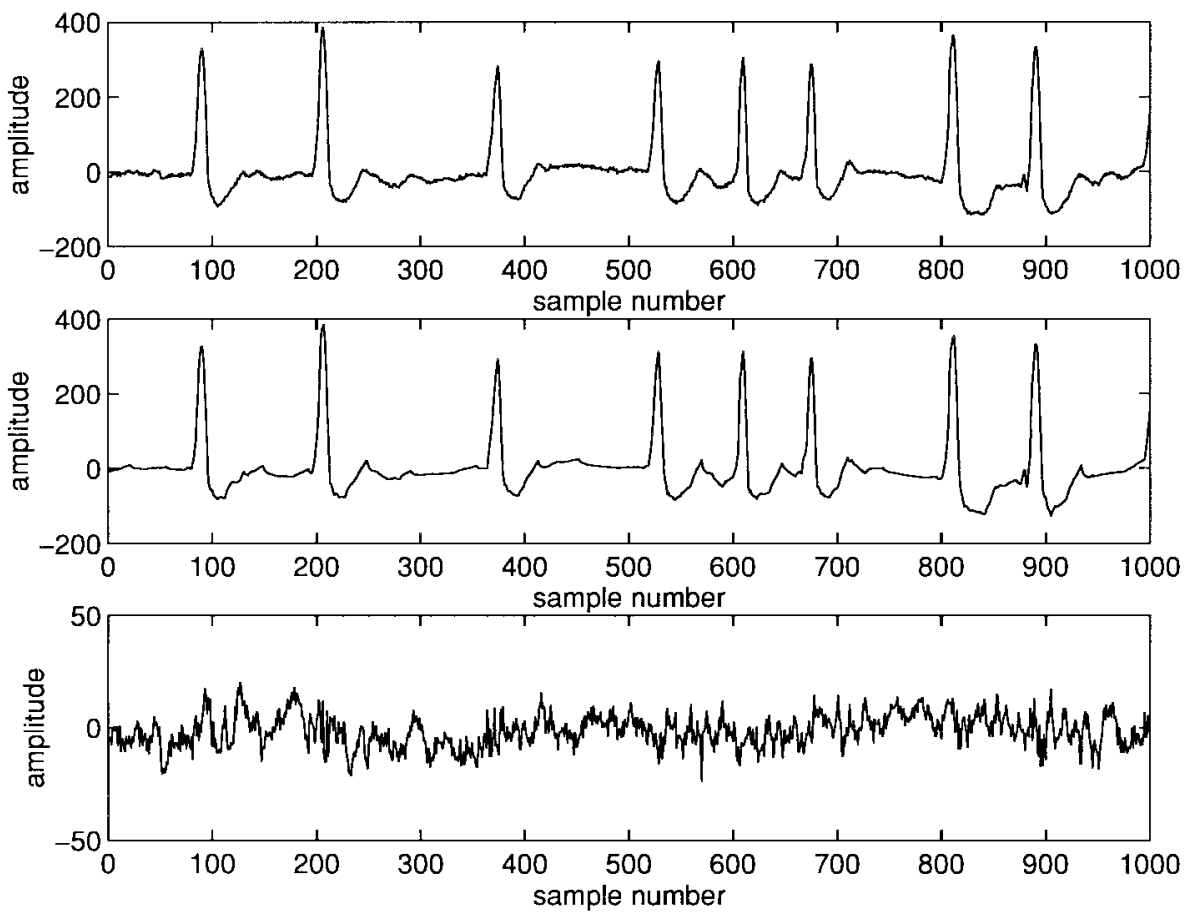

Fig. 6. Results of our technique on one subject. (a) Original ECG, (b) reconstructed signal, and (c) reconstruction error.

(a)

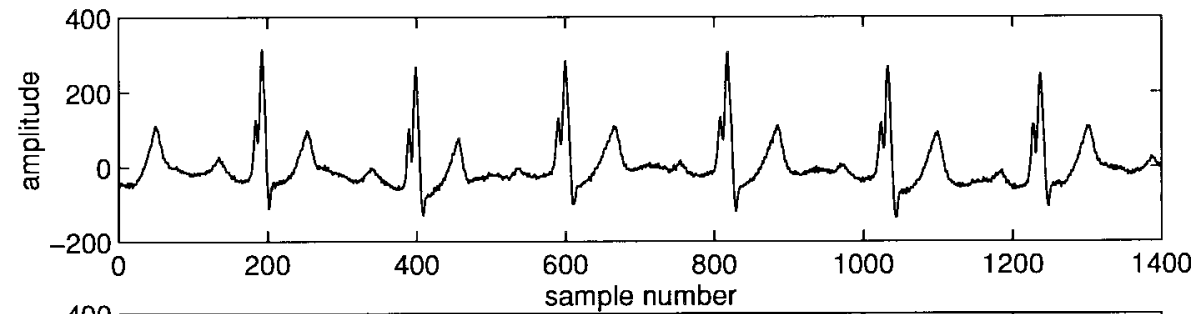

(b)
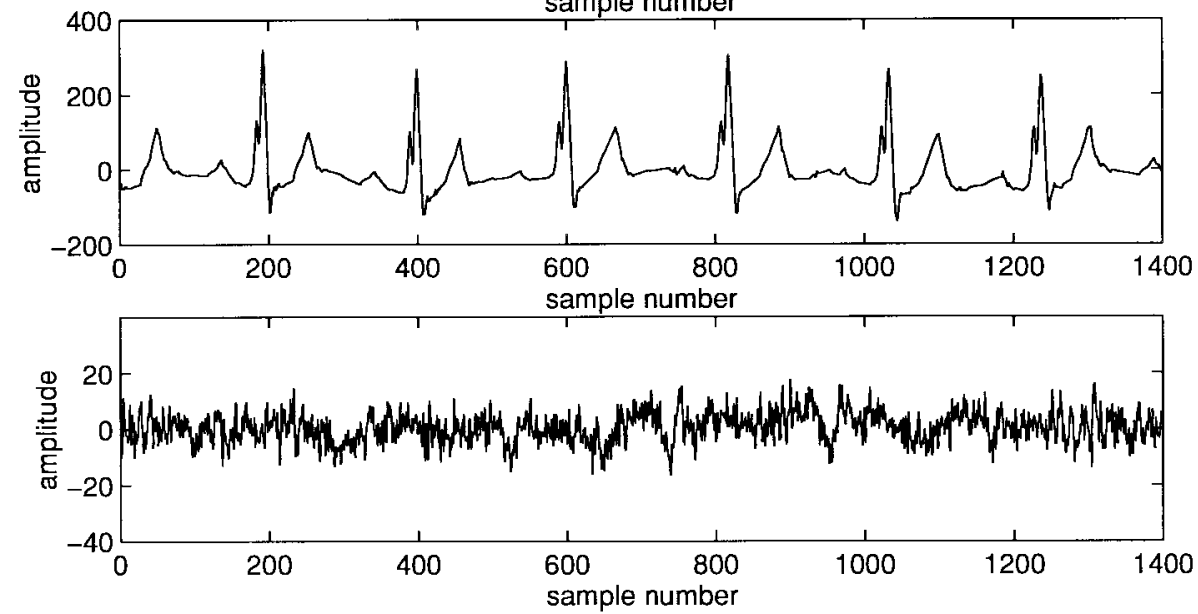

Fig. 7. Results of our technique on another subject. (a) Original ECG, (b) reconstructed signal, and (c) reconstruction error.

where $N$ is the total number of samples, and $x_{o}(i)$ and $x_{r}(i)$ are the $i$ th samples of original and reconstructed ECG, respectively. Since NRMSE is only an average measure, it alone cannot adequately quantify the performance of an ECG compression algorithm. This is because the QRS complex of the ECG carries significant morphological information, and error in this region must not be excessive. NRMSE does not give any idea about how the error is dispersed within any cycle.
Since the distribution of error within a cycle is also important in determining the clinical acceptability of the reconstructed data, we find out the maximum error and determine its position within each cycle.

\section{B. Normalized Maximum Amplitude Error (NMAE)}

The maximum amplitude of reconstruction error in each cycle is normalized by the dynamic range of the signal. The 
(a)

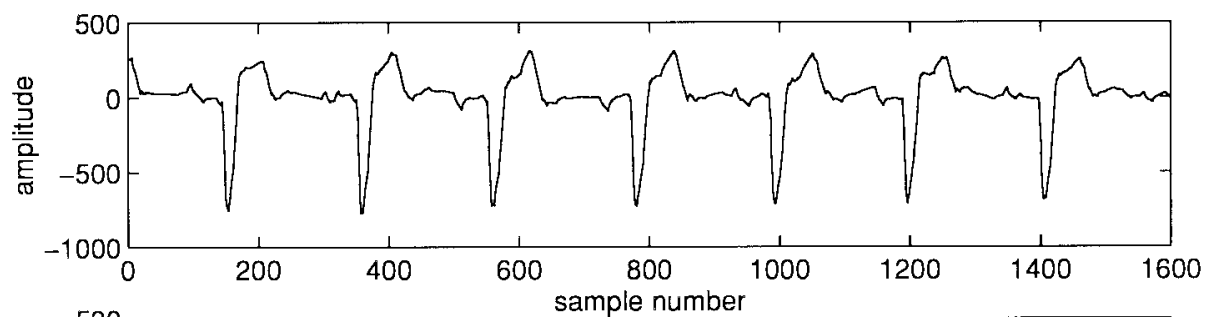

(b)

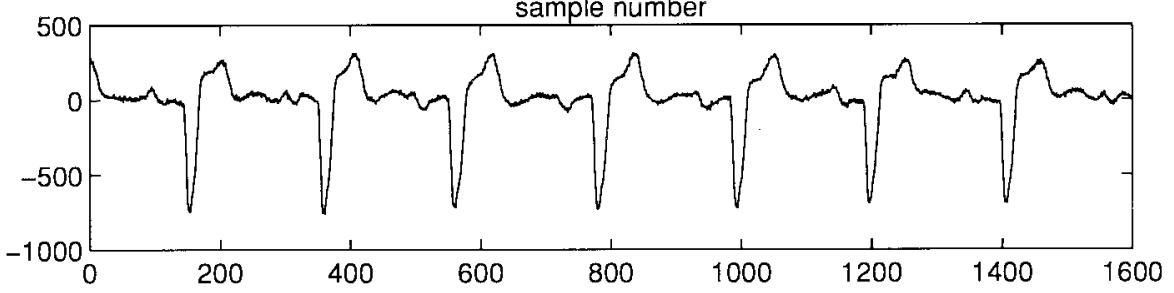

(c)

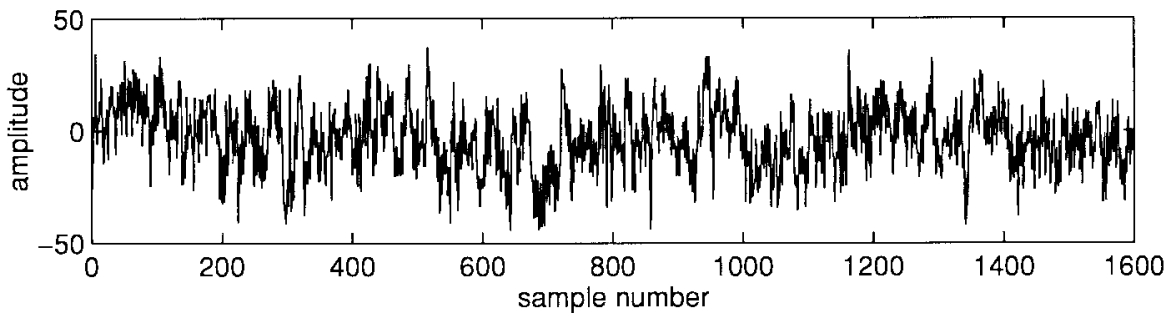

Fig. 8. Results of our technique on subject 3. (a) Original ECG, (b) reconstructed signal, and (c) reconstruction error.

expression for NMAE for the $i$ th cycle is

$$
\mathrm{NMAE}_{i}=\frac{\max \left|\mathbf{X}_{o i}-\mathbf{X}_{r i}\right|}{\max \mathbf{X}_{o i}-\min \mathbf{X}_{o i}} .
$$

The mean NMAE for a subject is obtained by averaging over all the cycles.

\section{Position of Maximal Reconstruction Errors}

It is interesting that the proposed approach entails nearly uniform distribution of error across the cycle. Besides, the maximum error in any cycle does not lie in the QRS region. Fig. 9 shows the positions of maximum errors in different cycles for a subject, plotted against the cycle number. The technique has performed well for different types of ECG waveforms due to:

1) period and amplitude normalization;

2) capability of wavelets to adapt to changes in morphology;

3) application of LP on wavelet coefficients across cycles rather than on the consecutive samples or wavelet coefficients of a cycle.

Table I gives the performance figures for five different subjects. It can be seen that the bit rate achieved varies from 135-225. The method proposed is elegant. It does not require any a priori knowledge of the ECG waveform. The significant advantage of the method is that the generally diagnostically vital QRS complexes are recovered with a fidelity as good as the other regions. In addition, the maximum error never occurred in the QRS regions, even while achieving a good CR and an overall error within limits. The distribution of reconstruction error is almost uniform, and thus the morphology of all the components are preserved. Most of the previous authors have

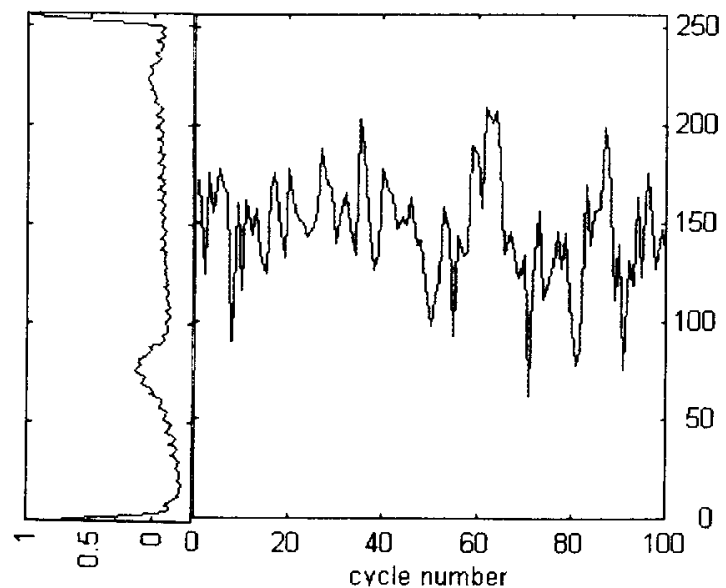

Fig. 9. Position within cycle of peak reconstruction error, plotted as a function of beat number, for one of the subjects. The PAN ECG beat, superimposed along the $y$-axis, aids in identifying the location with respect to the QRS complex.

not achieved this, with the exception of Philips [25]. Since the signal amplitude levels in the QRS region are very high, the QRS complex has been reconstructed with less relative error, than the other regions of the ECG. Thus the performance of the method can be considered good because it retains more clinically relevant information with high fidelity. This is because in wavelet based decomposition, multiresolution analysis is performed and the localized high-frequency region is reconstructed well, as the wavelet coefficients contributing to the QRS regions are unaltered (except for quantization loss). Thus, all important morphological information is retained. Since the beats considered are R-wave to R-wave (R-R), and the periods are transmitted, and since the wavelet bases 
TABLE I

Performance Figures for Five of the Subjects

\begin{tabular}{r|r|r|r}
\hline $\mathrm{CR}$ & Bit Rate & NRMSE\% & NMAE\% \\
\hline 16.76 & 178.9 & 10.07 & 5.03 \\
19.1 & 157.1 & 11.27 & 9.77 \\
15.24 & 196.8 & 11.73 & 5.22 \\
22.3 & 134.5 & 13.34 & 6.81 \\
13.4 & 223.8 & 9.89 & 4.96 \\
\hline
\end{tabular}

are time-localized, all rhythm information is also preserved. Normalization (PAN) of ECG cycles leads to LP that performs better, than is the case with nonnormalized cycles.

The computational complexity of the technique, while being comparable to those of [11] and [25], is higher than most methods. Thus, currently, the technique can be utilized for off-line applications such as patient databases and medical education systems. However, one can conceive of alternate, faster techniques for period normalization, in which case, with suitable dedicated hardware for normalization and DWT, and parallelization of possible steps (such as LP and amplitude normalization), real-time implementation of this technique may become possible.

In unusual circumstances, if the residuals are very high for an odd cycle, prediction can be avoided and the actual amplitudes of the top $N_{R}$ wavelet coefficients of that cycle can be sent. However, in the case of the database we used, this situation did not occur. Nonetheless, in order to evaluate the technique in such circumstances, we tested all our data with only this PAN-DWT-truncation technique. The expression for $\mathrm{CR}$ in this case reduces to

$$
\mathrm{CR}=\frac{\alpha_{o} \sum_{i=1}^{N_{T}} T_{i}}{N_{T}\left[\alpha_{a}+\alpha_{p}+N_{R}\left(\beta_{a}+\beta_{p}\right)\right]}
$$

where $\alpha_{o}, \alpha_{a}, \alpha_{p}, \beta_{a}$, and $\beta_{p}$ refer to the number of bits required for transmitting original sample, scale factor difference, period difference, the amplitude of each wavelet coefficient, and the difference between the locations, respectively. The other symbols are the same as in (16). Applying only this variation, we could still obtain mean $\mathrm{CR}$ values of above ten. A rigorous evaluation of this technique has been done in [26]. This modified technique is infinitely adaptive in that it transmits the most significant coefficients of any beat irrespective of its similarity or otherwise with the rest of the data.

\section{CONCLUSION}

A novel scheme for high fidelity coding of ECG has been proposed. Handling R-R beats eliminates the ambiguity that normally arises in deciding the endpoints of a cycle whenever PQRST beats are used. The method, based on LP of chosen wavelet coefficients of PAN beats, performs well with both normal and pathological data. The normalization of beats has not been attempted or even conceived of by any other earlier work, even where each beat is taken as an unit, or intercycle correlation is exploited [9], [10]. This preprocessing converts the ECG data into a near-cyclostationary sequence and enables the uniform choice of wavelet coefficients to be retained in each beat, and hence, their prediction. The earlier wavelet based compression schemes have not attempted this [17], [18]. With the proposed technique, a mean transmission rate of 180 $\mathrm{b} / \mathrm{s}$ has been achieved (for the data tested) with no compromise on the fidelity of reconstruction. It exploits both interbeat and intrabeat correlations. Further, the clinically more significant QRS complexes are coded with an error equal to that in the other regions in each cardiac cycle.

\section{ACKNOWLEDGMENT}

The authors would like to thank Prof. B. N. Gangadhar, National Institute of Mental Health and Neurosciences, Bangalore, India, for providing the ECG database; Prof. T. Chacko, Chairman, Foreign Languages Section, for copy editing this manuscript; the reviewers for improving the precision of their presentation; and to A. Bavisi for his help in manuscript preparation.

\section{REFERENCES}

[1] J. R. Cox, F. M. Nolle, H. A. Fozzard, and G. C. Oliver, "AZTEC: A preprocessing scheme for real time ECG rhythm analysis," IEEE Trans. Biomed. Eng., vol. BME-15, pp. 128-129, 1968.

[2] J. P. Abenstein and W. J. Tompkins, "A new data-reduction algorithm for real-time ECG rhythm analysis," IEEE Trans. Biomed. Eng., vol. 29 , pp. $43-48,1982$.

[3] M. Ishijima, S. B. Shin, G. H. Hostetter, and J. Sklansky, "Scan-along polygonal approximation for data compression of electrocardiograms," IEEE Trans. Biomed. Eng., vol. BME-30, pp. 723-729, 1983.

[4] N. Ahmed, P. J. Milne, and S. G. Harris, "Electrocardiographic data compression via orthogonal transforms," IEEE Trans. Biomed. Eng., vol. BME-22, pp. 484-487, 1975.

[5] G. P. Fragakis, G. Papakonstantinou, and S. G. Tzafestas, "A fast Walsh transform-based data compression multimicroprocessor system: Application to ECG signals," Math. Comput. Simulation, vol. 27, pp. 491-502, 1985

[6] B. R. S. Reddy and I. S. N. Murthy, "ECG data compression using Fourier descriptors," IEEE Trans. Biomed. Eng., vol. BME-33, pp. 428-434, 1986.

[7] R. Degani, G. Bortolan, and R. Murolo, "Karhunen-Loeve coding of ECG signals," Comput. Cardiol., 1991.

[8] M. E. Womble, J. S. Halliday, S. K. Mitter, M. C. Lancaster, and J. H. Triebwasser, "Data compression for storing and transmitting ECGs/VCG's," Proc. IEEE, vol. 65, pp. 702-706, 1977.

[9] G. Nave and A. Cohen, "ECG compression using long-term prediction," IEEE Trans. Biomed. Eng., vol. 40, pp. 877-885, 1993.

[10] P. S. Hamilton and W. J. Tompkins, "Compression of ambulatory ECG by average beat subtraction and residual differencing," IEEE Trans. Biomed. Eng., vol. 38, pp. 253-259, 1991.

[11] B. Madhukar and I. S. N. Murthy, "ECG data compression by modeling," Comput. Biomed. Res., vol. 26, pp. 310-317, 1993.

[12] J. A. Cadzow and T. T. Hwang, "Signal representation: An efficient procedure," IEEE Trans. Acoust., Speech, Signal Processing, vol. ASSP25, pp. 461-465, 1977.

[13] P. S. Hamilton and W. J. Tompkins, "Quantitative investigation of QRS detection rules using the MIT/BIH arrhythmia database," IEEE Trans. Biomed. Eng., vol. BME-33, pp. 1157-1165, 1986.

[14] P. P. Vaidyanathan, Multirate Systems and Filter Banks. Englewood Cliffs, NJ: Prentice Hall, 1993.

[15] W. Philips and G. De Jonghe, "Data compression of ECG's by highdegree polynomial approximation," IEEE Trans. Biomed. Eng., vol. 39, pp. 330-337, 1992.

[16] L. Senhadji, J. J. Bellanger, G. Carrault, and J. L. Coatrieux, "Wavelet analysis of ECG signals," in Proc. Ann. Int. Conf. IEEE Eng., Med. Biol. Soc., 1990, vol. 12, pp. 811-812.

[17] N. V. Thakor, Y. Sun, H. Rix, and P. Caminal, "Multiwave: A waveletbased ECG data compression algorithm," IEICE Trans. Inform. Syst, vol. E76-D, pp. 1462-1469, 1993. 
[18] B. Bradie, "Wavelet-packet based compression of single lead ECG," IEEE Trans. Biomed. Eng., vol. 43, pp. 493-501, 1996.

[19] C. K. Chui, An Introduction to Wavelets. New York: Academic, 1992.

[20] D. E. Newland, Random Vibrations, Spectral and Wavelet Analysis. New York: Wiley, 1993.

[21] I. Daubechies, "Orthonormal bases of compactly supported wavelets," Commn. Pure Appl. Math., vol. 41, pp. 909-996, 1988.

[22] S. Mallat, "A theory for multiresolution signal decomposition: The wavelet representation," IEEE Trans. Pattern Anal. Machine Intell., vol. 11, pp. 674-693, 1989.

[23] U. E. Ruttiman and H. V. Pipberger, "Compression of the ECG by prediction or interpolation and entropy encoding," IEEE Trans. Biomed. Eng., vol. BME-26, pp. 613-623, 1979.

[24] J. Makhoul, "Linear prediction-A tutorial review," Proc. IEEE, vol 63, pp. 561-580, 1975.

[25] W. Philips, "ECG compression by time warped polynomials," IEEE Trans. Biomed. Eng., vol. 40, pp. 1095-1401, 1993.

[26] S. Saha, "ECG compression algorithms after period and amplitude normalization," Master of Science thesis, Indian Inst. Sci., Bangalore, India, 1996.

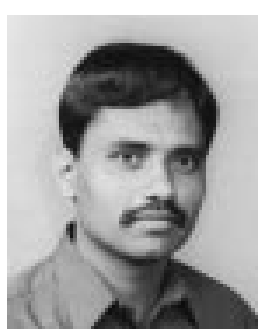

A. G. Ramakrishnan (S'81-M'82-SM'96) received the Ph.D. degree from the Indian Institute of Technology, Madras, India.

After working with BPL India and Fetzer Institute, Kalamazoo, MI, USA, he joined the Indian Institute of Science, Bangalore, India, in 1994 as Assistant Professor of Electrical Engineering. His interests include signal processing and instrumentation in medicine.

Dr. Ramakrishnan has received Thangam Vasudevan Award (Indian Association of Biomedical Scientists), Sir Andrew Watt Kay Young Researcher's Award (Royal College of Physicians and Surgeons, Glasgow, Scotland), and Young Investigator Award (Department of Science and Technology, Government of India). He is a member of the IEEE EMBS and SP societies, faculty advisor of the EMBS student Club, and the Editor of ENGMEDNEWS, the bimonthly newsletter of the Biomedical Engineering Society of India.

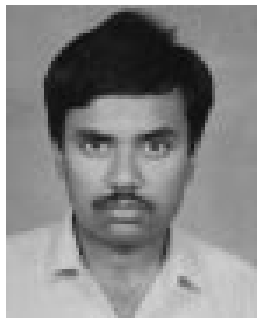

Supratim Saha (S'97) was born in Delhi, India. $\mathrm{He}$ received the B.Tech. (Honors) degree from the Indian Institute of Technology, Kharagpur, India, in 1993. He received the M.S. degree from the Indian Institute of Science, Bangalore, India, in 1996.

From July 1993 to April 1994, he was with INDAL, Calcutta, India, where he worked as an Instrumentation Engineer. Since August 1994, he has been with the Indian Institute of Science, Bangalore, India. He is currently working as a Project Associate in the Department of Electrical Communication Engineering at the same Institute. His current areas of interest are statistical signal processing with applications to blind equalization and discrete multitone systems. 